\title{
A Universal Process: How Mind and Matter Seem to Work
}

\author{
Osman Yaşar \\ State University of New York, the College at Brockport, Brockport, New York, USA
}

\section{Email address:}

oyasar@brockport.edu

\section{To cite this article:}

Osman Yaşar. A Universal Process: How Mind and Matter Seem to Work. Science Discovery. Vol. 3, No. 6, 2015, pp. 76-81. doi: 10.11648/j.sd.20150306.16

\begin{abstract}
There are scientific reports suggesting striking similarities between the structures of networked systems, ranging from the tiny brain cells to atoms, to the Internet, and all the way up to even the galaxies. It is further argued that the similarities might be due to the existence of a universal natural growth process. At the microscopic level we do not yet know what that mechanism might be, however, we do have some significant clues at the macroscopic level, which do indicate that both mind (a network of the brain cells) and matter (i.e., network of atoms, molecules, planets, and galaxies) operate similarly. This article attempts to briefly explain such similarities using an abstract growth process and a structural representation along with some general concepts from computing, cognitive and natural sciences. This operational structure is well aligned with the latest empirical research on cognitive psychology and neuroscience. Technology tools whose pedagogical use is also aligned with our operational structure of the mind have been found to consistently increase student engagement and achievement in secondary schools. The same growth process and structural representation seem to describe the behavior and growth of the matter in the universe in many ways we can all relate to. Our interdisciplinary experience and analysis of analogies in different fields offer support to reports by the physicists and biologists about existence of a universal growth mechanism.
\end{abstract}

Keywords: Computational Modeling and Simulation, Pedagogy, Cognitive Psychology

\section{Introduction}

Recent evidence and scientific reports argue that the structure of a brain cell is strikingly similar to the structure of the universe [1-4]. For instance, a study published in Nature's Scientific Reports argued that the resemblance between the two could mean that the universe and the brain may have evolved in the same way [5]. In short, a universal mechanism may be in place in the fabric of the cosmos that guides the growth and development of systems large and small. From the electrical firing between brain cells, the growth of social and computer networks, and even to the expansion of galaxies, this process seems to show itself repeatedly [2]. At the microscopic level we do not yet know what that process might be and what fundamental principles might be driving events at that level but we do have some significant clues at the macroscopic level, which indicate that matter and mind (a cumulative and cooperative work of the brain cells) might be operating similarly.

This article attempts to merge some general concepts and fundamental principles from computing, cognitive and natural sciences to explain briefly a potential cause of these similarities using an abstract representation (Fig. 1). Another purpose of this paper is to bring to the attention of educators the existence of a pedagogical technology tool whose successful implementation and operational mechanism provide empirical data to support our abstract view of how the mind learns. Positive and consistent findings from the years of using such pedagogical technology tools in teaching and learning have been already documented by the author and his colleagues in [6-17] but we will offer a brief introduction and provide relevant references to readers interested in details.

Figure 1 might help us understand in general terms how matter and mind seem to form and grow. First we will operationally define every packet of distinguishable stuff around us - be it information or matter - as a model. An apple, an orange, an atom, a neuron, and an electron can all be considered models of matter. A word, a sentence, a summary, and a book can also be considered as models of information. Every model, just like the colored circles in Fig. 1, hides its internal details from the outer world. And this can even be applied to the process in Fig. 1 since we are using it as an abstract representation to illustrate how matter and mind might be behaving. So, a model simplifies things by hiding what actually might be happening in it in greater details. This is an important characteristic that we will emphasize in later sections in the con- 
text of matter and information. Finally, models either unite to form bigger models or break down into sub models as illustrated by deductive (top-down) and inductive (bottom-up) arrows in Fig. 1.

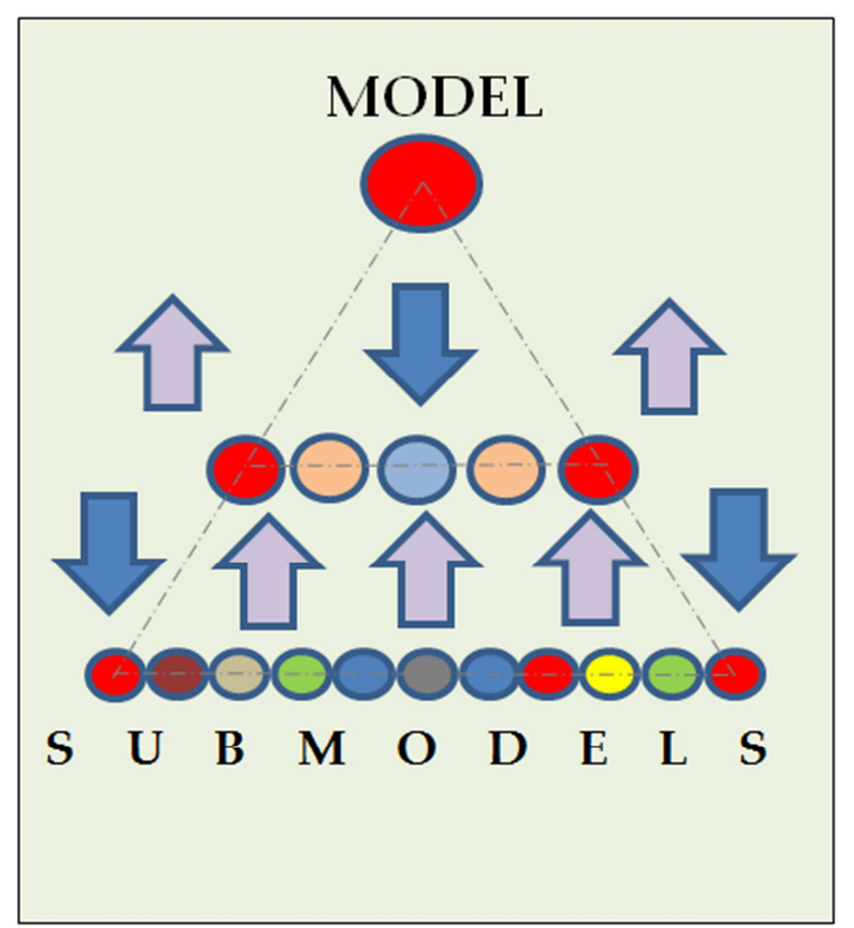

Figure 1. An abstract representation to illustrate how matter and mind work.

\section{A Universal Process}

Based on the operational definitions in the previous section, we assume that: a) matter and mind respectively contain packets of energy and information that conceal their internal elements from outside observations, and b) matter and information packets break down deductively to smaller ones or unite inductively to form bigger ones as a result of a trial and error process driven by various conditions and rules of engagement. Below, we will expand on these assumptions.

If we apply the structure of Fig. 1 to the matter in our universe, the highest level would contain a single model in the form of the whole space. The level below would contain multiple sub models in the form of galaxies and the levels below would continue the same trend down to stars, visible matter, molecules, atoms, protons, neutrons, electrons, and other smaller fundamental particles. The bottom level would then contain the tiniest elementary particles. The space and time scales for interactions (e.g., collisions) among models at a level will depend on the physical sizes and intensity of the models at that level - the higher the level, the larger the scales. For example, the scales at the intergalactic level are many orders of magnitude higher than those at the interatomic level. When the difference is too big between two levels, they end up concealing their internal elements from each other. An observer from the space will miss what is going on in a football game here on the earth. With the same token, we miss what is happening inside a computer chip. While such concealment (abstraction) benefits us as we explain below, we have managed to pierce it through using technologies, such as telescopes and microscopes, to either scale up or down representations of the events occurring at levels above and below ours. So, while at the microscopic level the matter is made of atoms and other smaller particles, at the macroscopic level we miss these details and see only their unions and generalized forms. As a result, one can say that the nature has a tendency to present itself in abstract forms and luckily we benefit from this. If we saw atomic details of the matter all the time, we would lose focus and waste a lifetime following tiny particles.

If we apply the same structure to the information that is processed by our minds, the lowest level would contain the tiniest details. As we move up inductively, each level would contain unions of the details from the levels below it - the higher the level, the more general the information. The space-time scales discussed earlier would also apply here. The tiniest information packet (e.g., letter A) would take less space and time for processing and representation than larger packets (e.g., a word "Apple") above its level, and this trend would continue all the way to a sentence, paragraph, chapter, book, etc. What is critical to note is that our brain hardware possess structures and protocols that match information's hierarchical dynamics as described. The way we both store and process information involves distributive (top-down) and associative (bottom-up) characteristics [18] as shown in Fig.1.

In contemporary times, we are bombarded with information from all directions yet we find a way through, using abstraction skills to rise above paralyzing details and countless thoughts; the higher the level of abstraction, the wider its applicability and validity [19]. Abstraction is a form of inductive thinking and it helps our cognitive development by simplifying, categorizing, and registering key information and knowledge for quicker retrieval and processing [20]. Abstraction aids us in our personal and professional lives. Dijkstra, an early pioneer in programming, regarded abstraction as the most vital activity of a competent programmer [21]. We all use abstract thinking every day. For example, most of us do not care to see how cooks prepare our meals. Those who actually do and visit restaurant kitchens soon abandon this behavior. And, those who would continuously operate at the increasingly smaller level of details (the bottom level in Fig. 1) would hardly be able to function in society due to the attention they are paying to details. This myopic view of the world could even cause burdens such as delayed action, indecisiveness, or inaction, as dramatized by Shakespeare through the Hamlet character [22].

In reality, we grow up moving back and forth, as needed, between very detailed and very generalized information. In the process of doing so, the details our brain is able to register and store, the hierarchical connections it establishes between them, and the generalizations, conclusions, and principles it forms through abstractions all build over time like a pyramid (or a tree) structure and an operational mechanism that we call brain software, or mind as often referenced [19]. The brain attempts to interpret every new concept and information that it encounters in terms of previously registered models - objects, 
faces, scenarios, etc. [20]. As we grow older, the relationships among registered information lead to interplay of various combinations and scenarios that end up inductively clustering related details into conclusions, generalizations, and more inclusive models of information. For example, before the age of 8-10 months babies do not grasp that food or a pacifier falling from their mouths actually falls down due to gravity into a third dimension; they think that such objects just disappear from existence. But, as a result of what-if scenarios (i.e., simulations) played by their brains and the action of connecting related dots over time babies eventually conclude about existence of a downward dimension [23-24].

Perhaps, one could claim that we may have developed abstract thinking skills as a result of a survival concern for having limited resources (i.e., time, memory, attention). For example, our tendency to summarize and generalize information - before we permanently store it - might be a strategy to overcome limited storage capacity. Such tendency can also help us to shield ourselves from details that have no practical value for survival. Having very limited ability for multitasking might be the reason for our minds' tendency to bring closure to a subject so we can move on and spend our energy on other issues. However, recent findings in neuroscience indicate that it is not just the limited capacity of our brain but also its distributed structure that drives a bi-directional flow of information resulting in such tendencies that benefit us. Both the storage and processing of information involve a synchronized distributed participation of all neurons in related regions of the brain [18].

Computing experts would know from parallel computing that management and utilization of a distributed hardware necessitates scatter and gather type functionalities in software. And, that seems to be what is happening in the brain as well [25]. A newborn's brain contains 100 billion neurons that get connected to each other in various ways. Information is stored into the memory in the form of specific patterns of neurons placed on a pathway that are fired together [18]. The number and strength of such pathways improve the storage and retrieval of information. A memory can be a combination of previously formed memories, each of which might also involve a vast network of concepts and details mapped onto the brain's neural network. When new information arrives, it lights up all related neurons and pathways in a distributive process that is similar to the top-down action in Fig. 1, whereas a model is broken up into related pieces. On the other hand, retrieving a memory is a reassembly of its original pattern of neurons and pathways in an associative process that is similar to the bottom-up action in Fig. 1. Remembering is often regarded as an act of creative re-imagination and what is retrieved is probably not the original pattern but one with some holes or extra bits. Some neuroscientists recently argue that there is no distinction between the act of remembering and thinking in the brain [18]. This reminds us of another analogy within the computing world. In early days of computing, data and instructions were stored distinctly but when the distinction was removed, computing became much easier and more powerful.

\section{How Matter and Mind May Have Evolved}

Matter seems to have formed as a result of packaging (modeling) out of a homogeneous energy field as the space expanded and the temperature dropped shortly after the big bang [26]. We still do not know in greater details how an elementary particle - a particle model whose substructure is unknown - gains its mass and shields its content from the rest of the energy field that gives birth to it, but we believe that the interactions and collisions between such particles (e.g., quarks, electrons, etc.) led to an interplay of various combinations and scenarios, which must have ended up uniting related ones later into bigger structures gradually over time. First, quarks combined to make protons and neutrons, then they combined to make nuclei, and finally atoms came into being once temperature cooled enough for electrons' kinetic energy to escape electromagnetic attraction by protons. Heterogeneity increased further through gravitational attraction as clots of matter grew to form stars and those lumps of matter grew to form galaxies. The universe keeps expanding, thereby cooling further to perhaps collapse the matter into a big crunch, but until then - which may be followed by another big bang - it appears that the matter in our universe has also been experiencing an opposite top-down process, as seen in Fig. 1, by which bigger models (stars, molecules, atoms, etc.) have broken down to smaller sub models, both within our earthly scale and the galactic scales (e.g., supernovas). This two-way evolution still seems to be continuing.

Matter becomes distinguishable to us not only because of its heterogeneity at the source level in the form of distinct objects but also as a result of the information from these objects being received by our senses and processed by our brains. The growth of our brain hardware and software is a bit complex and many things can go wrong during a lifespan. Normally, at birth, the circuitry at the inner part of the brain is up and running to manage vital and involuntary functions (e.g., breathing, heartbeat, and some degree of sound and visual tracking), but the outer part (cerebral cortex) takes some time to be ready for voluntary actions (e.g., conscious thought, information storage and processing) [25]. Actually, the majority of neurons that a human is born with are contained within this thin cortex that separates humans from other animals. While only a few neurons develop during adulthood, we can take comfort that mental growth is not solely based on the number of neurons in the brain, but rather the increasing complexity of the connections between them. Other key factors include the functionality each neuron or groups of neurons assume, the size they grow into, and the placement in different parts of the brain that they migrate towards. Even more important is the number of inter-neuronal connections, which are estimated to be near 100 trillion. New neural connections are being made all the time as we learn new things, in fact these connections constitute the definition of learning, and the existing connections are strengthened, weakened, or even eliminated if not revisited often enough. Genetics plays only a partial role determining the growth of the brain, as there are not enough genes on the 
human chromosome to code for the placement of billions of neurons and trillions of connections [25]. This luckily leaves plenty of room for the brain (and the mind) to continue growing as a result of one's free will, experience, and environment. The next sections will offer some recommendations and generalizations from our discussion as well as a modeling and simulation technology tool to accelerate a mindful process we all have inherited.

\section{Modeling and Simulation Process}

Our view of how the mind learns has additional backing from the scientific methodology for research, which is the same process that is described in Fig. 1. Scientists often start with a model, such as an assumption, a concept or a theory, based on their existing knowledge, facts, and information. They, then, move deductively into sub models and related details to investigate their models further and gather additional data. They test various what-if scenarios by changing relevant parameters, environmental conditions, and characteristics of the sub models and their relationships. Based on new findings, facts, and relationships, they inductively build a new model or revise the original one. This two-way modeling, testing, what-if scenarios, decision-making, and change process is repeated as resources permit until there is confidence in the model's validity and reliability. If the newly arrived model is the same as the old one, then the initial assumption would prove valid. If it is different, then we often say that a conceptual change has taken place.

A new framework [27] for the U.S. national K-12 science standards suggests that students learn in a more meaningful and robust way if they are engaged in activities closely resembling the way scientists think and work. While the process of acting and experience learning like a scientist is rarely offered as a method of instruction, recent technologies are now making it possible to put in the hands of students the tools used by scientists. With its increasing power and easy use, computational modeling and simulation technology (C-MST) has become a common tool. It was at the disposal of scientists for many decades, but today it is commonly found in schools and on personal mobile devices.

Over the past decade, more than 700 pre-service and in-service teachers have been trained on the methodology and principles of C-MST. The findings indicate that C-MST tools are effective in both teaching and learning of science and mathematics [6-17]. Since students learn well with the help of C-MST tools, one can infer that these tools must be feeding the mind the way it actually works as suggested here. C-MST tools enabled students to conduct complex science experiments using readymade models, which eliminated the need for any prerequisite knowledge in math, computing, and science. Students were exposed to science concepts in an incremental way as they demonstrated better skills and higher interest. Such a deductive approach created an effective remedy to raise interest in mathematics and science among thousands of students in various settings in both urban and suburban school districts. We would have been content and declared success, however, students who became proficient in the use of these tools took it a step further by creating their own models and conducting controlled simulations with them. Some curious and able students wondered about how these tools worked and conducted even free experiments which eventually led to discovery of underlying mathematical, computational and scientific principles either on their own or often with help from teacher or peers. This final step was nothing but the inductive process that has been mentioned here. Their overall experience was nothing but how scientists do their work.

\section{Discussion}

It appears that the brain's distributed neural structure enables it to store, process, and retrieve information in a scatter/gather fashion that matches the distributive and associative characteristics of matter and information [18-25]. Such a harmony among basic gears of a system offers many potential benefits but we may not be utilizing it to the fullest extent possible. There are many lessons to extract from the universality of such a structure and dynamism.

While the matter in our universe seems to have been consistently following a bidirectional evolution [26], our minds have followed separate paths and many often have resisted change. Sticking longer than necessary to our misconceptions and preconceptions is an example of that. If cycling back and forth is a norm in our universe, then we should strike a balance between opposing ends - e.g., skepticism and certainty, details and general, and deductive and inductive teaching - but instead we often advocate for only one of these ends, stick to it for too long, and create camps with opposition and tension in between, especially in education and pedagogy. There is a major problem in education that could be eased if such tension is removed. For example, students form many misconceptions and preconceptions before they reach the school age, and this has been a major challenge to teaching and learning [20]. It is important to provide them with skills and tools and teach them to examine their knowledge, assumptions, concepts and beliefs by breaking them down to their constitutive details, test them under various conditions, and finally rebuild those concepts inductively using newly collected data and facts. This matches the recommendations of recent U.S. national K-12 standards to teach early graders decomposition (distributive) and abstraction (associative) skills as part of the computational thinking (CT) skill set [28]. Scientists have already been using CT skills, and it is time to teach everyone, as there are now easy-to-use modeling and simulation tools to foster them at young age.

Technology has already been helping us extend our perspectives - both within and outside our scale - beyond what we normally gain through our brain's biological computations and simulations [24]. A few examples include still images and videos by cameras, microscopes and telescopes as well as interactions with and observations of others across the globe through transportation, communication, and social-media technologies. There is now even a greater opportunity, through modeling and simulation tools, to improve the capacity of our mind through simulations of systems in a wide range of both 
space and time scales, all the way from elementary particles to galaxies. These tools are already being used and proven effective in education. Their use in teaching lends itself to a natural pedagogy [6-17] that resonates with how the mind works for the reasons we have described earlier. However, full utilization of such tools to foster inquiry, creativity, and critical thinking would only be realized if their principles of operation are well understood. The benefit would be multifold. Learners may be able to transfer the acquired knowledge to their experience with other tools, concepts, and settings. Perhaps, even more importantly, by correlating the process of modeling and simulation to the way how matter and mind work, they may be tempted to develop higher-level metacognitive skills and undertake self-evaluations by gauging how they address their assumptions and conceptions and engage in both deductive and inductive thinking as often and efficiently as possible. We may be able to accelerate the evolution of our minds along these lines and gain a more universal awareness that is mindful of almost everything around us and free of many, if not all, unfounded assumptions, preconceptions and misconceptions.

\section{Conclusions \& Recommendations}

This work is a culmination of many years of research in engineering, physics, computer and cognitive sciences as well as teaching at college level and secondary schools. It was inspired by the evidence of student learning as a result of using technology tools in secondary school classrooms. The study of the effectiveness of such tools led to discovering their pedagogical nature [6-17]. As collaboration with cognitive psychologists and neuroscientists [18-25] increased our understanding of how the mind learns, we discovered the resemblance between the way the mind stores and retrieves information and the way modeling and simulation tools are used in scientific research and education. Reports from the scientific community suggesting similarities between structures of the universe and of the brain cells then encouraged us to examine if the process in Fig. 1 can also describe how the matter behaves. Putting together our experience from three domains (computing, cognitive science, and natural sciences), we arrived at a conclusion that the process in Fig. 1 describes the operation of both matter and mind.

By describing how fundamental the process of modeling and simulation is, we wanted to encourage its wide-spread use in education. Additionally, by arguing the existence of a few plausible universal principles as common characteristics of matter and mind, we wanted to set a discourse among researchers and educators from different fields. Even if readers may object to what we view as proofs for the validity of these principles as summarized below, we hope that they may still find them interesting as opinion:

- Discrete formation and distinguishability are keys to the operation of matter and mind.

- Emergence of a discrete form (model) results from an inductive process driven by a collective behavior of sub models based on relationships and bonds between them.

- Evolution of matter and mind seems to be following a bi-directional (deductive and inductive) process.

\section{Acknowledgements}

This work has resulted from work supported by the National Science Foundation (NSF) funds via Grants \#1136332, $\# 0942569$ and \#0226962. We thank to faculty and teachers who contributed to the study of the effectiveness of computational modeling and simulation in teaching and learning.

\section{References}

[1] Rundle, M. "Physicists Find Evidence That the Universe is a Giant Brian.” Huffington Post UK, November 27, 2012.

[2] Ghose, T. "Universe, Brain, and Internet: Growth patterns Similar in Large \& Small Networks, Computer Study Suggests." Huffington Post UK. Also, "Universe Grows Like a Giant Brain.” Life Science, November 26, 2012.

[3] Miller, M. (2006). Neuronal Network. Brandeis Univ. http://www.visualcomplexity.com/vc/project.cfm?id=307. Retrieved October 15, 2015.

[4] Millennium Simulation. Science Daily. http://www.sciencedaily.com/releases/2005/06/050604061156. htm. June 4, 2005.

[5] Krioukov, D., Kitsak, M., Sinkovits, R. S., Rideout, D., Meyer, D. and Boguna, M. "Network Cosmology." Scientific Reports, Nature, Article 793. http://www.nature.com/srep/2012/121113/srep00793/full/srep 00793.html. November 16, 2012.

[6] Yaşar, O., Veronesi, P., Maliekal, J. and Little, L. (2015). "Computational Pedagogical Content Knowledge (CPACK)." In D. Slykhuis \& G. Marks (Eds.), Proceedings of Society for Information Technology \& Teacher Education International Conference 2015 (pp. 3514-3521).

[7] Yaşar, O. and Maliekal, J. (2014). "Computational Pedagogy." IEEE Comp Sci \& Eng, 16 (3), 2014; 78-88.

[8] Yaşar, O. (2014). “A Pedagogical Approach to Teaching Computing Principles in the Context of Modeling and Simulations." J. Computing Teachers, Winter Issue.

[9] Yaşar, O., Maliekal, J., Veronesi, P. and Little, L. (2014). “An Interdisciplinary Approach to Professional Development of Math, Science, and Technology Teachers." J. Comp. in Math and Science Teaching, 33 (3), 2014; 349-374.

[10] Yaşar, O. (2013). "Computational Math, Science, and Technology (C-MST) Approach to General Education Courses." $J$. Computational Science Education, Vol. 4 (1), 2013; 2-10.

[11] Yaşar, O. (2013). "Teaching Science through Computation." Int J. Science, Technology and Society, Vol. 1 (1), 2013; 9-18.

[12] Yaşar, O., Maliekal, J., Little, L. and Jones, D. (2006). "A Computational Technology Approach to Education." IEEE $J$. Comp. in Sci. \& Eng., 8 (3), 2006; 76-81.

[13] Yaşar, O., Little, L., Tuzun, R., Rajasethupathy, K., Maliekal, J. and Tahar, M. (2006). "Computational Math, Science, and Technology (C-MST)." Lecture Notes in Computer Science, 3992, 2006; 169-176. 
[14] Yaşar, O. (2004). "C-MST Pedagogical Approach to Math and Science Education." Lecture Notes in Computer Science, 3045, 2004; 807-816.

[15] Yaşar, O. and Landau, R. (2003). "Elements of Computational Science and Engineering Education," SIAM Review, 45 (4), 2003; 787-805

[16] Yaşar, O. (2001). "Computational Science Education: Standards, Learning Outcomes and Assessment," Lecture Notes in Computer Science, 2073, 2001; 1159-1169.

[17] Yaşar, O., Rajasethupathy, K., Tuzun, R., McCoy, A. and Harkin, J. (2000). "A New Perspective on Computational Science Education.” IEEE Comp. in Sci \& Eng, 5(2), 2000; 74-79.

[18] Brown, P. C. Roediger, H. L. and McDaniel, M. A. (2014). Make it Stick. The Belknap Press of Harvard University.

[19] Tenenbaum, J. B., Kemp, C., Griffiths, T. L. and Goodman, N. D. (2011). "How to Grow a Mind: Statistics, Structure, and Abstraction." Science, 331, 2011; 1279-1285.

[20] Bransford, J., Brown, A. \& Cocking, R. (2000). How People Learn. National Academy Press, Washington, D.C.
[21] Armoni, M. (2013). "On Teaching Abstraction to Computer Science Novices." J. Comp in Math \& Science Teaching, 32 (3), $2013 ; 265-284$.

[22] Shakespeare, W. (1982). Hamlet. Ed. Harold Jenkins. London.

[23] Mooney, C. G. (2013). An Introduction to Dewey, Montessori, Erikson, Piaget, and Vygotsky. Redleaf Press: St. Paul, MN.

[24] Montague, R. (2006). How We Make Decisions. Plume Books: New York.

[25] Restak, R. (2001). The Secret Life of the Brain. The Dana Press: New York.

[26] Hawking, S. (1988). A Brief History of Time. Random House.

[27] A Framework for K-12 Science Education. The National Academies Press, Washington, D.C. http://www.nap.edu.

[28] Wing, J. M. (2006). "Computational Thinking," Comm. ACM, 49 (3), 2006; 33-35. 